\title{
Radial Quadrature for Multiexponential Integrands
}

\author{
PETER M. W. GILL, SIU-HUNG CHIEN \\ School of Chemistry, University of Nottingham, Nottingham NG7 2RD, United Kingdom
}

Received 30 August 2002; Accepted 30 August 2002

\begin{abstract}
We introduce a Gaussian quadrature, based on the polynomials that are orthogonal with respect to the weight function $\ln ^{2} x$ on the interval $[0,1]$, which is suitable for the evaluation of radial integrals. The quadrature is exact if the non-Jacobian part of the integrand is a linear combination of a geometric sequence of exponential functions. We find that the new scheme is a useful alternative to existing approaches, particularly for integrands that exhibit multiexponential behavior.
\end{abstract}

(C) 2003 Wiley Periodicals, Inc. J Comput Chem 24: 732-740, 2003

Key words: radial quadrature; DFT quadrature; logarithmic weight function; Gaussian quadrature

\section{Introduction}

We live in a three-dimensional world, and problems in chemical physics therefore often demand the evaluation of three-dimensional integrals:

$$
I=\int_{-\infty}^{+\infty} \int_{-\infty}^{+\infty} \int_{-\infty}^{+\infty} F(x, y, z) d x d y d z
$$

If the physical problem possesses a natural origin, it is generally convenient to transform eq. (1) into spherical polar coordinates to obtain the radial integral

$$
I=\int_{0}^{\infty} r^{2} f(r) d r
$$

where the Jacobian factor $r^{2}$ arises from the coordinate transformation and

$f(r)=\int_{0}^{\pi} \int_{0}^{2 \pi} F(r \sin \theta \sin \phi, r \sin \theta \cos \phi, r \cos \theta) \sin \theta d \phi d \theta$

is the spherical average of $F(x, y, z)$. If the integrals (2) and (3) can be performed in closed form, it is usually beneficial to do so. However, in general, this is not possible and one must turn instead to approximations. If detailed mathematical properties of $F(x, y$, $z$ ) or $f(r)$ are known, it may be possible to design approximations that specifically exploit these. More often, however, only global properties may be known, for example that $f(r)$ is analytic almost everywhere, decays exponentially, and so forth. In such cases, there is no option but to utilize generic methods. It is these that are our present concern.

The generic numerical approach to integrals such as (2) and (3) is to suppose that the integrand can be approximated accurately by expansion in a suitable basis set and develop a quadrature rule from this. For the angular integral (3), which corresponds to a proper integration over the surface of a sphere, the spherical harmonics form a natural basis and this leads to a family of $n$-point quadrature formulae:

$$
\int_{0}^{n} \int_{0}^{2 \pi} h(\theta, \phi) \sin \theta d \phi d \theta \approx \sum_{i=1}^{n} w_{i}^{\theta} h\left(\theta_{i}, \phi_{i}\right)
$$

that has been investigated by several workers. ${ }^{1-6}$ Such formulae are exact if $h(\theta, \phi)$ is a linear combination of spherical harmonics whose orders do not exceed $L \approx V(3 n)-1$. The improper radial integral (2) is more problematic. We would like to approximate it by the $n$-point quadrature:

$$
I \approx \sum_{i=1}^{n} w_{i} f\left(r_{i}\right)
$$

but what is a good basis for the determination of the roots $r_{i}$ and weights $w_{i}$ ?

In the next section, we review some of the approaches that have been suggested previously. The section New Radial Grid intro-

Correspondence to: P. M. W. Gill 
duces a new approach, specifically tailored to functions $f(r)$ with components that decay on different length scales. The section Roots and Weights compares the roots and weights of the old and new schemes, and the section Conditions for Exactness discusses the types of integrands for which each scheme is exact. Finally, in the section Examples, we assess relative accuracies for a selection of examples.

\section{Previous Radial Grids}

The classical approach to the radial quadrature problem is to combine the transformation ${ }^{7}$

$$
\begin{gathered}
r=x R \\
I=R^{3} \int_{0}^{\infty} x^{2} f(r) d x
\end{gathered}
$$

with Gauss-Laguerre quadrature ${ }^{8}$ to obtain the Laguerre grid:

$$
\begin{gathered}
r_{i}=x_{i} R \\
w_{i}=\frac{x_{i}^{3} \exp \left(x_{i}\right)}{(n+1)^{2}\left[L_{n+1}\left(x_{i}\right)\right]^{2}} R^{3} \\
x_{i}=i \text { th zero of } L_{n}(x)
\end{gathered}
$$

where $L_{n}(x)$ is the $n$th Laguerre polynomial. ${ }^{8}$

In 1988, Becke $^{9}$ suggested that the transformation

$$
\begin{gathered}
r=\frac{1+x}{1-x} R \\
I=2 R^{3} \int_{-1}^{+1} \frac{(1+x)^{2}}{(1-x)^{4}} f(r) d x
\end{gathered}
$$

be combined with Chebyshev quadrature of the second kind ${ }^{8}$ to obtain the Becke grid:

$$
\begin{gathered}
r_{i}=\frac{1+x_{i}}{1-x_{i}} R \\
w_{i}=\frac{2 \pi}{n+1} \frac{\left(1+x_{i}\right)^{5 / 2}}{\left(1-x_{i}\right)^{7 / 2}} R^{3} \\
x_{i}=\cos \left(\frac{i \pi}{n+1}\right)
\end{gathered}
$$

In 1993, Murray et al. ${ }^{10}$ combined the transformation

$$
r=\frac{x^{2}}{(1-x)^{2}} R
$$

$$
I=2 R^{3} \int_{0}^{1} \frac{x^{5}}{(1-x)^{7}} f(r) d x
$$

with the Euler-Maclaurin summation formula ${ }^{8}$ (which, in this context, is equivalent to the extended trapezoidal rule $^{8}$ ) to obtain the Handy grid:

$$
r_{i}=\frac{x_{i}^{2}}{\left(1-x_{i}\right)^{2}} R
$$

$$
w_{i}=\frac{2 x_{i}^{5}}{(n+1)\left(1-x_{i}\right)^{7}} R^{3}
$$

$$
x_{i}=\frac{i}{n+1}
$$

In 1995, Treutler and Ahlrichs studied some of the weaknesses of the Laguerre, Becke, and Handy quadratures and decided instead to combine ${ }^{11}$ the transformation

$$
\begin{array}{r}
r=-R \frac{(1+x)^{\alpha}}{\ln 2} \ln \left(\frac{1-x}{2}\right) \\
I=\left(\frac{R}{\ln 2}\right)^{3} \int_{-1}^{+1}(1+x)^{3 \alpha}\left[\frac{1}{1-x} \ln ^{2}\left(\frac{1-x}{2}\right)\right. \\
\left.-\frac{\alpha}{1+x} \ln ^{3}\left(\frac{1-x}{2}\right)\right] f(r) d x
\end{array}
$$

with Chebyshev quadrature of the second kind to obtain the Ahlrichs grid:

$$
\begin{gathered}
r_{i}=-R \frac{\left(1+x_{i}\right)^{\alpha}}{\ln 2} \ln \left(\frac{1-x_{i}}{2}\right) \\
w_{i}=\frac{\pi}{n+1} \frac{\left(1+x_{i}\right)^{3 \alpha}}{\ln ^{3} 2}\left[\sqrt{\frac{1+x_{i}}{1-x_{i}}} \ln ^{2}\left(\frac{1-x_{i}}{2}\right)\right. \\
\left.-\alpha \sqrt{\frac{1-x_{i}}{1+x_{i}}} \ln ^{3}\left(\frac{1-x_{i}}{2}\right)\right] R^{3} \\
x_{i}=\cos \left(\frac{i \pi}{n+1}\right)
\end{gathered}
$$

On the basis of extensive atomic calculations, these authors recommended $\alpha=0.6$ and concluded that the Ahlrichs grid is more accurate than those of Becke and Handy.

In 1996, Mura and Knowles suggested ${ }^{12}$ that the transformation

$$
r=-R \ln \left(1-x^{3}\right)
$$

$$
I=3 R^{3} \int_{0}^{1} \frac{x^{2} \ln ^{2}\left(1-x^{3}\right)}{1-x^{3}} f(r) d x
$$


be combined with the Euler-Maclaurin formula to obtain the Knowles grid:

$$
\begin{gathered}
r_{i}=-R \ln \left(1-x_{i}^{3}\right) \\
w_{i}=\frac{3 x_{i}^{2} \ln ^{2}\left(1-x_{i}^{3}\right)}{(n+1)\left(1-x_{i}^{3}\right)} R^{3} \\
x_{i}=\frac{i}{n+1}
\end{gathered}
$$

Mura and Knowles studied its accuracy in atomic applications and found it slightly better than Ahlrichs' grid (especially for small $n$ ) and considerably better than Handy's.

None of the quadrature schemes discussed above are perfect, and each can be criticized on both aesthetic and practical grounds. The Becke, Handy, Ahlrichs, and Knowles schemes all map the semi-infinite domain $[0, \infty)$ onto a finite domain and then apply either Chebyshev or trapezoidal quadrature. However, the combinations of mapping and quadrature are not convincingly justified and, as we will show in the section Conditions for Exactness, each yields a grid that is exact for functions $f(r)$ that rarely arise in chemical physics. Laguerre quadrature is more direct, but the end result is the same.

In the next section, we take the opposite approach, deliberately constructing a quadrature that is exact for an important class of integrands. By proceeding in this way, we ensure that the resulting scheme will be ideal for at least one important type of integral.

\section{New Radial Grid}

The Gaussian quadrature rule of the form

$$
\int_{0}^{1} g(x) \ln ^{2} x d x \approx \sum_{i=1}^{n} a_{i} g\left(x_{i}\right)
$$

is related to the system of polynomials $Q_{n}(x)$ orthogonal on the interval $[0,1]$ with respect to the weight function $\ln ^{2} x$. This system does not appear to have been studied previously ${ }^{13}$ but, by inverting the Cholesky triangle of the associated Gram matrix, ${ }^{14}$ we have constructed $^{15}$ the $Q_{n}(x)$ for $1 \leq n \leq 27$. The associated roots $\left(x_{i}\right)$ and weights $\left(a_{i}\right)$ for $n=1-6,8,10,15,20$ are listed in Table 1.

For the radial quadrature problem, we then propose that the transformation

$$
\begin{gathered}
r=-R \ln x \\
I=R^{3} \int_{0}^{1} x^{-1} f(r) \ln ^{2} x d x
\end{gathered}
$$

be combined with the "log-squared" quadrature (31) to obtain the MultiExp grid:

$$
\begin{aligned}
r_{i} & =-R \ln x_{i} \\
w_{i} & =\left(a_{i} / x_{i}\right) R^{3}
\end{aligned}
$$

\section{Roots and Weights}

It is informative to compare the roots and weights from the various quadrature schemes. There is some ambiguity in this because the roots given by eqs. $(8,13,18,23,28,34)$ are proportional to arbitrary scale factors $R$ (not necessarily the same) and the weights given by eqs. $(9,14,19,24,29,35)$ are proportional to $R^{3}$. Therefore, for the purposes of comparison, we choose the $R$ values so that the middle root of each quadrature is unity. We call the resulting values the standardized roots and weights and they are listed, for $n=1,3,5,7,9$, and 11, in Tables 2 and 3. The weights in Table 3 are given in scientific notation, the power of ten being shown in parentheses.

We note first that the Ahlrichs and Knowles grids are strikingly alike, especially near the middle of the grid. This is initially surprising because their derivations are superficially very different. However, because both were specifically optimized for use in atomic quadrature, their apparent similarity is perhaps not entirely unexpected.

The most obvious difference between the sets of roots in Table 2 is their spread about the median value of unity. We illustrate this in the case of 11-point quadrature by plotting the logs of the roots in Figure 1. Our standardization ensures that the curves coincide at the middle root $\left(\log r_{6} \equiv 0\right)$, but the Handy and Becke grids extend further out, and the Ahlrichs and Knowles grids extend further in than the others. The Laguerre and MultiExp grids have the narrowest range of the six.

Table 3 shows analogous behavior for the weights. There is an infallible correlation between the size of a root and its weight: small roots have very small weights, large roots have very large weights. Thus, although the middle standardized weight is always close to unity, the spread about this varies greatly from one grid to another. The greatest variation, and the largest values, are found in the Handy grids (the $n=11$ weights span 12 orders of magnitude, and the largest is greater than $10^{6}$ ) and the least variation in the Laguerre and MultiExp grids (the $n=11$ weights span only six orders of magnitude). The smallest weights arise in the Ahlrichs and Knowles grids (the least of the $n=11$ weights is less than $10^{-6}$ ).

We have examined these observations analytically by determining the dependence of the innermost and outermost roots and weights on $n$. The results, which are given in Table 4, show that the Ahlrichs and Knowles grids run from small roots (with very small weights) to moderately large roots. The Becke and Handy grids run from moderately small roots to large roots (with very large weights). The MultiExp grid runs from moderately small roots to moderately large roots.

In practical applications, unless $f(r)$ is singular at the origin, small roots with very small weights will usually make negligible contributions to the quadrature. Similarly, unless $f(r)$ decays very slowly, large roots with very large weights will also contribute negligibly. Such roots can, of course, be systematically neglected within computer programs, but we agree with Mura and Knowles that it is preferable to use more appropriate grids from the outset. ${ }^{12}$ All things being equal, this philosophy predisposes us toward grids 
Table 1. Roots and Weights for $n$-Point Quadrature on $[0,1]$ with the Weight Function $\ln ^{2} x$.

\begin{tabular}{|c|c|c|c|c|c|}
\hline$n$ & $x_{i}$ & $a_{i}$ & $n$ & $x_{i}$ & $a_{i}$ \\
\hline 1 & 0.1250000000 & 2.0000000000 & 15 & 0.0031568454 & 0.3253137565 \\
\hline \multirow[t]{2}{*}{2} & 0.0598509925 & 1.6691361082 & & 0.0214217428 & 0.3961243562 \\
\hline & 0.4536625210 & 0.3308638918 & & 0.0567152146 & 0.3593522229 \\
\hline \multirow[t]{3}{*}{3} & 0.0362633111 & 1.3638303836 & & 0.1082790024 & 0.2926536989 \\
\hline & 0.2731486024 & 0.5658154596 & & 0.1744898369 & 0.2219588508 \\
\hline & 0.6537110896 & 0.0703541567 & & 0.2530501060 & 0.1582926857 \\
\hline \multirow[t]{4}{*}{4} & 0.0246451318 & 1.1330156422 & & 0.3411169468 & 0.1061466261 \\
\hline & 0.1831933310 & 0.6612166786 & & 0.4354309056 & 0.0665501730 \\
\hline & 0.4610171077 & 0.1857929500 & & 0.5324530702 & 0.0385929320 \\
\hline & 0.7655906466 & 0.0199747293 & & 0.6285097879 & 0.0203475527 \\
\hline \multirow[t]{5}{*}{5} & 0.0179624485 & 0.9588537970 & & 0.7199410768 & 0.0094938596 \\
\hline & 0.1317184306 & 0.6830020585 & & 0.8032477673 & 0.0037511449 \\
\hline & 0.3395971926 & 0.2815660272 & & 0.8752323216 & 0.0011607732 \\
\hline & 0.5945982935 & 0.0695856412 & & 0.9331298792 & 0.0002398218 \\
\hline & 0.8320575996 & 0.0069924762 & & 0.9747402975 & 0.0000215456 \\
\hline \multirow[t]{6}{*}{6} & 0.0137303290 & 0.8247373524 & 20 & 0.0019241239 & 0.2308490189 \\
\hline & 0.0994431475 & 0.6701662035 & & 0.0128189043 & 0.3027886725 \\
\hline & 0.2596678762 & 0.3457959549 & & 0.0338360585 & 0.2986810329 \\
\hline & 0.4685229897 & 0.1269936018 & & 0.0647886177 & 0.2678766357 \\
\hline & 0.6874245835 & 0.0294555188 & & 0.1051527594 & 0.2274158666 \\
\hline & 0.8742037763 & 0.0028513686 & & 0.1541448603 & 0.1852655647 \\
\hline \multirow[t]{8}{*}{8} & 0.0088308098 & 0.6343476124 & & 0.2107591088 & 0.1455612505 \\
\hline & 0.0626470137 & 0.6078905783 & & 0.2737989508 & 0.1104305753 \\
\hline & 0.1653937470 & 0.4036485580 & & 0.3419087328 & 0.0808087449 \\
\hline & 0.3076475309 & 0.2184654657 & & 0.4136071132 & 0.0568766276 \\
\hline & 0.4738811643 & 0.0959319490 & & 0.4873224017 & 0.0383323430 \\
\hline & 0.6449256028 & 0.0320637571 & & 0.5614294486 & 0.0245782243 \\
\hline & 0.8005576159 & 0.0069997943 & & 0.6342874604 & 0.0148581882 \\
\hline & 0.9222020825 & 0.0006522851 & & 0.7042779985 & 0.0083614715 \\
\hline \multirow[t]{10}{*}{10} & 0.0061869147 & 0.5075100632 & & 0.7698423653 & 0.0043003770 \\
\hline & 0.0431849645 & 0.5377370883 & & 0.8295175821 & 0.0019659640 \\
\hline & 0.1143932978 & 0.4101581499 & & 0.8819702175 & 0.0007640520 \\
\hline & 0.2157443263 & 0.2686821571 & & 0.9260275322 & 0.0002333787 \\
\hline & 0.3400163758 & 0.1544536152 & & 0.9607063554 & 0.0000477502 \\
\hline & 0.4777530668 & 0.0768964762 & & 0.9852482390 & 0.0000042614 \\
\hline & 0.6181540046 & 0.0319269841 & & & \\
\hline & 0.7500277506 & 0.0102578456 & & & \\
\hline & 0.8627655156 & 0.0021782820 & & & \\
\hline & 0.9472975116 & 0.0001993384 & & & \\
\hline
\end{tabular}

like Laguerre and MultiExp, but we must first ascertain whether or not they are sufficiently accurate to be useful.

\section{Conditions for Exactness}

In the last section, we compared the six quadrature schemes by visual comparison of their roots and weights. This is informative but gives little insight into the type of integrands for which each quadrature is accurate. Unfortunately, this question is not well defined so, in this section, we ask: for what set of integrands is each quadrature exact?

In order to study this, one must know the types of integrand for which Gauss-Laguerre, Chebyshev, and trapezoidal rules are exact. The Gauss-Laguerre and Chebyshev rules

$$
\begin{gathered}
\int_{0}^{\infty} g(x) \exp (-x) d x \approx \sum_{i=1}^{n} a_{i} g\left(x_{i}\right) \\
\int_{-1}^{+1} g(x) \sqrt{1-x^{2}} d x \approx \sum_{i=1}^{n} a_{i} g\left(x_{i}\right)
\end{gathered}
$$

are exact if $g(x)$ is a polynomial $P_{2 n-1}(x)$ of degree $2 n-1$ or less. The extended trapezoidal rule

$$
\int_{0}^{1} g(x) d x \approx \frac{1}{n+1} \sum_{i=1}^{n} g\left(\frac{i}{n+1}\right)
$$


Table 2. Standardized Roots $\left(r_{i}\right)$ for Radial Quadrature.

\begin{tabular}{|c|c|c|c|c|c|c|}
\hline$n$ & Laguerre & Becke & Handy & Ahlrichs & Knowles & MultiExp \\
\hline 1 & 1.0000 & 1.0000 & 1.0000 & 1.0000 & 1.0000 & 1.0000 \\
\hline \multirow[t]{3}{*}{3} & 0.1812 & 0.1716 & 0.1111 & 0.1093 & 0.1179 & 0.3276 \\
\hline & 1.0000 & 1.0000 & 1.0000 & 1.0000 & 1.0000 & 1.0000 \\
\hline & 2.7416 & 5.8284 & 9.0000 & 3.8201 & 4.1036 & 2.5559 \\
\hline \multirow[t]{5}{*}{5} & 0.0733 & 0.0718 & 0.0400 & 0.0299 & 0.0348 & 0.1702 \\
\hline & 0.3930 & 0.3333 & 0.2500 & 0.2738 & 0.2826 & 0.4814 \\
\hline & 1.0000 & 1.0000 & 1.0000 & 1.0000 & 1.0000 & 1.0000 \\
\hline & 1.9702 & 3.0000 & 4.0000 & 2.5509 & 2.6316 & 1.8769 \\
\hline & 3.5148 & 13.9282 & 25.0000 & 5.6704 & 6.4735 & 3.7218 \\
\hline \multirow[t]{7}{*}{7} & 0.0394 & 0.0396 & 0.0204 & 0.0119 & 0.0146 & 0.1050 \\
\hline & 0.2095 & 0.1716 & 0.1111 & 0.1093 & 0.1179 & 0.2902 \\
\hline & 0.5240 & 0.4465 & 0.3600 & 0.3987 & 0.4057 & 0.5779 \\
\hline & 1.0000 & 1.0000 & 1.0000 & 1.0000 & 1.0000 & 1.0000 \\
\hline & 1.6697 & 2.2398 & 2.7778 & 2.0599 & 2.0961 & 1.6226 \\
\hline & 2.5986 & 5.8284 & 9.0000 & 3.8201 & 4.1036 & 2.6115 \\
\hline & 3.9580 & 25.2741 & 49.0000 & 6.9830 & 8.3009 & 4.6255 \\
\hline \multirow[t]{9}{*}{9} & 0.0245 & 0.0251 & 0.0123 & 0.0058 & 0.0075 & 0.0714 \\
\hline & 0.1301 & 0.1056 & 0.0625 & 0.0536 & 0.0602 & 0.1952 \\
\hline & 0.3232 & 0.2596 & 0.1837 & 0.1957 & 0.2050 & 0.3823 \\
\hline & 0.6098 & 0.5279 & 0.4444 & 0.4899 & 0.4953 & 0.6441 \\
\hline & 1.0000 & 1.0000 & 1.0000 & 1.0000 & 1.0000 & 1.0000 \\
\hline & 1.5106 & 1.8944 & 2.2500 & 1.8021 & 1.8224 & 1.4850 \\
\hline & 2.1702 & 3.8518 & 5.4444 & 3.0070 & 3.1459 & 2.1699 \\
\hline & 3.0353 & 9.4721 & 16.0000 & 4.8358 & 5.3728 & 3.2296 \\
\hline & 4.2505 & 39.8635 & 81.0000 & 7.9935 & 9.7778 & 5.3542 \\
\hline \multirow[t]{11}{*}{11} & 0.0168 & 0.0173 & 0.0083 & 0.0032 & 0.0043 & 0.0517 \\
\hline & 0.0886 & 0.0718 & 0.0400 & 0.0299 & 0.0348 & 0.1407 \\
\hline & 0.2193 & 0.1716 & 0.1111 & 0.1093 & 0.1179 & 0.2732 \\
\hline & 0.4116 & 0.3333 & 0.2500 & 0.2738 & 0.2826 & 0.4544 \\
\hline & 0.6697 & 0.5888 & 0.5102 & 0.5581 & 0.5623 & 0.6924 \\
\hline & 1.0000 & 1.0000 & 1.0000 & 1.0000 & 1.0000 & 1.0000 \\
\hline & 1.4123 & 1.6984 & 1.9600 & 1.6442 & 1.6570 & 1.3981 \\
\hline & 1.9217 & 3.0000 & 4.0000 & 2.5509 & 2.6316 & 1.9235 \\
\hline & 2.5538 & 5.8284 & 9.0000 & 3.8201 & 4.1036 & 2.6497 \\
\hline & 3.3579 & 13.9282 & 25.0000 & 5.6704 & 6.4735 & 3.7582 \\
\hline & 4.4604 & 57.6955 & 121.0000 & 8.8138 & 11.0145 & 5.9604 \\
\hline
\end{tabular}

is exact if $g(x)$ equals $L(x)$, a continuous function that is linear between the roots $x_{i}=i /(n+1)$ and which vanishes at $x=0$ and $x=1$. if

Using eq. (36), one finds that the Laguerre grid (8-10) is exact

$$
f(r)=r^{-2} \exp (-r / R) P_{2 n-1}(r)
$$

The grid also works well when eq. (39) is approximately true, but it becomes less satisfactory when $f(r)$ either decays more slowly than an exponential or contains multiple exponential components with significantly different rates of decay. ${ }^{16}$

The Chebyshev-based Becke grid is exact if

$$
\frac{(1+x)^{2}}{(1-x)^{4}} f(r) \frac{1}{\sqrt{1-x^{2}}}=P_{2 n-1}(x)
$$

which implies

$$
f(r)=\frac{1}{r^{3 / 2}(r+R)^{3}} P_{2 n-1}\left(\frac{r-R}{r+R}\right)
$$

In general, such $f(r)$ possess a $1 / r^{3 / 2}$ singularity at the origin and decay as $1 / r^{9 / 2}$ for large $r$.

The trapezoidal-based Handy grid is exact if

$$
\frac{x^{5}}{(1-x)^{7}} f(r)=L(x)
$$

which implies

$$
f(r)=\frac{1}{r^{5 / 2}(\sqrt{r}+\sqrt{R})^{2}} L\left(\frac{\sqrt{r}}{\sqrt{r}+\sqrt{R}}\right)
$$

In general, such $f(r)$ possess a $1 / r^{2}$ singularity at the origin and decay as $1 / r^{7 / 2}$ for large $r$. 
Table 3. Standardized Weights $\left(w_{i}\right)$ for Radial Quadrature.

\begin{tabular}{|c|c|c|c|c|c|c|}
\hline$n$ & Laguerre & Becke & Handy & Ahlrichs & Knowles & MultiExp \\
\hline 1 & $(+0) 2.72$ & $(+0) 3.14$ & $(+0) 4.00$ & $(+0) 3.21$ & $(+0) 3.21$ & $(+0) 1.78$ \\
\hline \multirow[t]{3}{*}{3} & $(-2) 1.54$ & $(-2) 1.12$ & $(-3) 3.66$ & $(-3) 4.17$ & $(-3) 4.96$ & $(-2) 4.92$ \\
\hline & $(+0) 1.20$ & $(+0) 1.57$ & $(+0) 2.00$ & $(+0) 1.60$ & $(+0) 1.60$ & $(-1) 9.48$ \\
\hline & $(+1) 1.83$ & $(+2) 4.40$ & $(+3) 1.94$ & $(+1) 6.59$ & $(+1) 9.20$ & $(+1) 1.72$ \\
\hline \multirow[t]{5}{*}{5} & $(-3) 1.01$ & $(-4) 7.75$ & (-4) 1.54 & $(-5) 8.58$ & $(-4) 1.26$ & $(-3) 6.67$ \\
\hline & $(-2) 7.04$ & $(-2) 4.48$ & $(-2) 2.34$ & $(-2) 3.27$ & $(-2) 3.45$ & $(-2) 9.29$ \\
\hline & $(-1) 7.70$ & $(+0) 1.05$ & $(+0) 1.33$ & $(+0) 1.07$ & $(+0) 1.07$ & $(-1) 6.58$ \\
\hline & $(+0) 4.66$ & $(+1) 3.26$ & $(+1) 9.60$ & $(+1) 1.39$ & $(+1) 1.64$ & $(+0) 4.12$ \\
\hline & $(+1) 2.48$ & $(+3) 5.66$ & $(+4) 3.75$ & $(+2) 1.47$ & $(+2) 2.59$ & $(+1) 4.24$ \\
\hline \multirow[t]{7}{*}{7} & $(-4) 1.57$ & $(-4) 1.27$ & $(-5) 1.94$ & $(-6) 5.44$ & $(-6) 9.42$ & $(-3) 1.55$ \\
\hline & $(-2) 1.05$ & $(-3) 5.61$ & $(-3) 1.83$ & $(-3) 2.09$ & $(-3) 2.48$ & $(-2) 1.96$ \\
\hline & $(-1) 1.07$ & $(-2) 7.57$ & $(-2) 4.98$ & $(-2) 6.74$ & $(-2) 6.86$ & $(-1) 1.16$ \\
\hline & $(-1) 5.66$ & $(-1) 7.85$ & $(+0) 1.00$ & $(-1) 8.02$ & $(-1) 8.02$ & $(-1) 5.07$ \\
\hline & $(+0) 2.19$ & $(+0) 9.55$ & $(+1) 2.29$ & $(+0) 5.75$ & $(+0) 6.38$ & $(+0) 2.01$ \\
\hline & $(+0) 7.41$ & $(+2) 2.20$ & $(+2) 9.72$ & $(+1) 3.30$ & $(+1) 4.60$ & $(+0) 8.83$ \\
\hline & $(+1) 2.69$ & $(+4) 3.31$ & $(+5) 2.69$ & $(+2) 2.22$ & $(+2) 4.49$ & $(+1) 7.08$ \\
\hline \multirow[t]{9}{*}{9} & $(-5) 3.80$ & $(-5) 3.21$ & $(-6) 4.18$ & $(-7) 6.40$ & $(-6) 1.26$ & $(-4) 4.83$ \\
\hline & $(-3) 2.51$ & $(-3) 1.26$ & $(-4) 3.05$ & $(-4) 2.46$ & $(-4) 3.28$ & $(-3) 5.87$ \\
\hline & $(-2) 2.49$ & $(-2) 1.36$ & $(-3) 5.90$ & $(-3) 7.97$ & $(-3) 8.73$ & $(-2) 3.25$ \\
\hline & $(-1) 1.25$ & $(-2) 9.72$ & $(-2) 7.32$ & $(-2) 9.38$ & $(-2) 9.42$ & $(-1) 1.26$ \\
\hline & $(-1) 4.47$ & $(-1) 6.28$ & $(-1) 8.00$ & $(-1) 6.42$ & $(-1) 6.42$ & $(-1) 4.13$ \\
\hline & $(+0) 1.32$ & $(+0) 4.49$ & $(+0) 9.49$ & $(+0) 3.18$ & $(+0) 3.43$ & $(+0) 1.25$ \\
\hline & $(+0) 3.53$ & $(+1) 4.44$ & $(+2) 1.54$ & $(+1) 1.32$ & $(+1) 1.66$ & $(+0) 3.89$ \\
\hline & $(+0) 9.22$ & (+2) 9.08 & (+3) 5.12 & (+1) 5.35 & $(+1) 8.51$ & (+1) 1.43 \\
\hline & $(+1) 2.73$ & $(+5) 1.29$ & $(+6) 1.18$ & $(+2) 2.88$ & $(+2) 6.42$ & $(+1) 9.96$ \\
\hline \multirow[t]{11}{*}{11} & $(-5) 1.21$ & $(-5) 1.05$ & $(-6) 1.23$ & $(-7) 1.11$ & $(-7) 2.44$ & $(-4) 1.83$ \\
\hline & $(-4) 7.93$ & $(-4) 3.88$ & $(-5) 7.68$ & $(-5) 4.29$ & $(-5) 6.31$ & $(-3) 2.18$ \\
\hline & $(-3) 7.74$ & $(-3) 3.74$ & $(-3) 1.22$ & $(-3) 1.39$ & $(-3) 1.65$ & $(-2) 1.16$ \\
\hline & $(-2) 3.80$ & $(-2) 2.24$ & $(-2) 1.17$ & $(-2) 1.64$ & $(-2) 1.73$ & $(-2) 4.29$ \\
\hline & $(-1) 1.31$ & $(-1) 1.11$ & $(-2) 9.11$ & $(-1) 1.11$ & $(-1) 1.11$ & $(-1) 1.30$ \\
\hline & $(-1) 3.69$ & $(-1) 5.24$ & $(-1) 6.67$ & $(-1) 5.35$ & $(-1) 5.35$ & $(-1) 3.48$ \\
\hline & $(-1) 9.12$ & $(+0) 2.66$ & $(+0) 5.16$ & $(+0) 2.06$ & $(+0) 2.18$ & $(-1) 8.87$ \\
\hline & $(+1) 2.09$ & $(+1) 1.63$ & $(+1) 4.80$ & $(+0) 6.93$ & $(+0) 8.19$ & $(+0) 2.25$ \\
\hline & $(+0) 4.61$ & $(+2) 1.47$ & $(+2) 6.48$ & $(+1) 2.20$ & $(+1) 3.07$ & $(+0) 6.11$ \\
\hline & $(+1) 1.04$ & $(+3) 2.83$ & $(+4) 1.88$ & $(+1) 7.36$ & $(+2) 1.29$ & $(+1) 2.02$ \\
\hline & (+1) 2.70 & $(+5) 3.89$ & (+6) 3.87 & (+2) 3.48 & $(+2) 8.31$ & (+2) 1.28 \\
\hline
\end{tabular}

Unfortunately, because the transformation (21) cannot be conveniently inverted, we have not been able to find the set of functions $f(r)$ for which the Ahlrichs grid is exact.

The trapezoidal-based Knowles grid is exact if

$$
\frac{x^{2} \ln ^{2}\left(1-x^{3}\right)}{1-x^{3}} f(r)=L(x)
$$

which implies

$$
f(r)=\frac{e^{-r / R}}{r^{2}\left(1-e^{-r / R}\right)^{2 / 3}} L\left[\left(1-e^{-r / R}\right)^{1 / 3}\right]
$$

In general, such $f(r)$ possess a $1 / r^{7 / 3}$ singularity at the origin and decay exponentially.

The MultiExp grid is exact if

$$
x^{-1} f(r)=P_{2 n-1}(x)
$$

which implies

$$
f(r)=\sum_{k=1}^{2 m} c_{k} \exp (-k r / R)
$$

Obviously, such $f(r)$ are linear combinations of exponential functions.

The accuracy with which a quadrature scheme estimates the value of an integral depends on how close the integrand is to the set of functions for which the quadrature is exact. Our principal interest lies in the development of efficient quadrature schemes for the types of integral that arise in atomic and molecular calculations using density functional theory. ${ }^{17}$ Because of shell structure, the radial electron density around an atom can usually be modeled well by a sum of exponential functions with a variety of decay constants. $^{18}$ It has previously been noted ${ }^{10,11}$ that such multiexponential character may limit the usefulness of the Laguerre grid, 


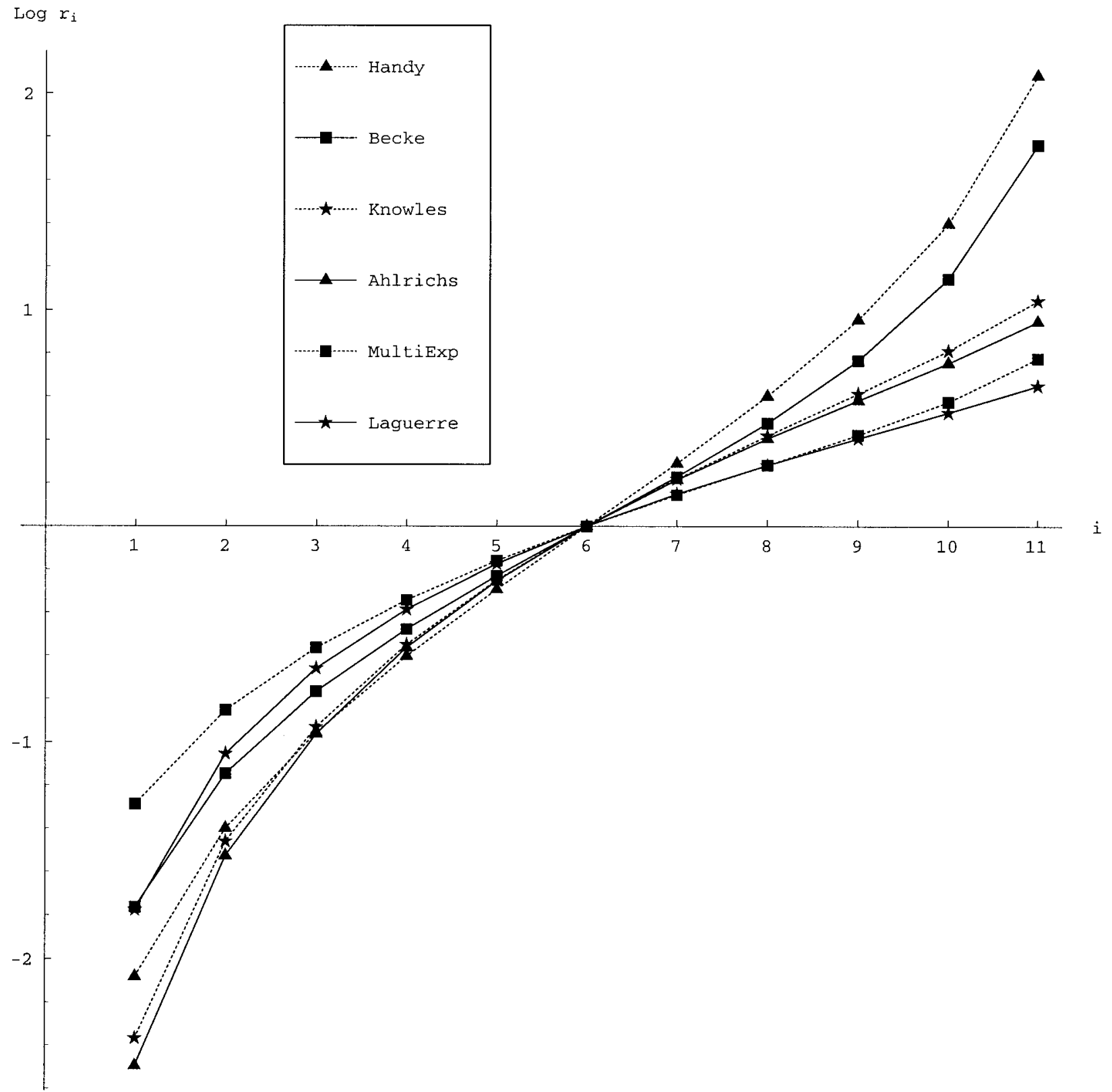

Figure 1. Logarithms (base 10) of the standardized roots for the 11-point Handy, Becke, Knowles, Ahlrichs, MultiExp, and Laguerre grids.

but it is clear that the MultiExp grid is well suited to such problems.

\section{Examples}

Whereas the theoretical results of the foregoing section are rigorous, the task of selecting specific numerical examples to illustrate quadrature accuracy is a vexed one, for one can always select examples to make a given scheme look as good, or as bad, as one wishes. For this reason, we advise the reader not to attach too much significance to the handful of examples that we discuss
Table 4. Innermost and Outermost Roots and Weights for $n$-Point Quadrature.

\begin{tabular}{llllll}
\hline & \multicolumn{2}{c}{ Innermost } & & \multicolumn{2}{c}{ Outermost } \\
\cline { 2 - 3 } \cline { 5 - 6 } & Root & Weight & & Root & Weight \\
\hline Laguerre & $O\left(n^{-1}\right)$ & $O\left(n^{-3}\right)$ & & $O(n)$ & $O\left(n^{3}\right)$ \\
Becke & $O\left(n^{-2}\right)$ & $O\left(n^{-6}\right)$ & & $O\left(n^{2}\right)$ & $O\left(n^{6}\right)$ \\
Handy & $O\left(n^{-2}\right)$ & $O\left(n^{-6}\right)$ & & $O\left(n^{2}\right)$ & $O\left(n^{6}\right)$ \\
Ahlrichs & $O\left(n^{-3.2}\right)$ & $O\left(n^{-9.6}\right)$ & & $O(\ln n)$ & $O\left(\ln ^{2} n\right)$ \\
Knowles & $O\left(n^{-3}\right)$ & $O\left(n^{-9}\right)$ & & $O(\ln n)$ & $O\left(\ln ^{2} n\right)$ \\
MultiExp & $O\left(n^{-2}\right)$ & $O\left(n^{-6}\right)$ & & $O(\ln n)$ & $O\left(\ln ^{2} n\right)$
\end{tabular}


Table 5. Quadrature Accuracy. ${ }^{\mathrm{a}}$

\begin{tabular}{|c|c|c|c|c|c|c|c|}
\hline$f(r)$ & $n$ & Laguerre & Becke & Handy & Ahlrichs & Knowles & MultiExp \\
\hline \multirow[t]{5}{*}{$\exp \left(-r^{2}\right)$} & 3 & 1.3 & 0.5 & 0.2 & 0.5 & 0.5 & 1.2 \\
\hline & 5 & 2.3 & 1.5 & 0.8 & 2.5 & 2.5 & 2.8 \\
\hline & 7 & 3.8 & 1.3 & 1.4 & 1.9 & 1.7 & 3.9 \\
\hline & 9 & 5.7 & 3.1 & 1.3 & 3.8 & 4.5 & 4.3 \\
\hline & 11 & 6.8 & 2.3 & 2.0 & 3.7 & 3.3 & 4.9 \\
\hline \multirow{5}{*}{$\begin{array}{l}\exp \left(-r^{2}\right)+10 \\
\quad \exp \left(-10 r^{2}\right)\end{array}$} & 3 & 2.2 & 0.8 & 0.4 & 1.1 & 1.0 & 2.2 \\
\hline & 5 & 1.5 & 2.2 & 1.3 & 1.5 & 1.6 & 2.4 \\
\hline & 7 & 2.7 & 1.5 & 2.2 & 1.7 & 2.0 & 3.1 \\
\hline & 9 & 3.3 & 2.5 & 1.5 & 2.5 & 2.5 & 4.3 \\
\hline & 11 & 4.9 & 2.5 & 2.4 & 3.2 & 3.7 & 4.8 \\
\hline \multirow{5}{*}{$\begin{array}{l}\exp \left(-r^{2}\right)+10 \\
\quad \exp \left(-10 r^{2}\right)+100 \\
\quad \exp \left(-100 r^{2}\right)\end{array}$} & 9 & 2.1 & 2.5 & 1.5 & 2.0 & 2.0 & 2.4 \\
\hline & 11 & 3.0 & 2.5 & 2.4 & 2.4 & 2.3 & 3.1 \\
\hline & 13 & 3.7 & 3.4 & 2.3 & 3.0 & 2.8 & 3.9 \\
\hline & 15 & 3.8 & 3.6 & 2.9 & 3.3 & 4.9 & 4.8 \\
\hline & 17 & 4.3 & 4.1 & 3.5 & 3.5 & 3.9 & 5.6 \\
\hline \multirow{5}{*}{$\begin{array}{l}\text { He atom density HF/6- } \\
\quad 311 \mathrm{G}\end{array}$} & 9 & 3.4 & 2.3 & 2.3 & 2.4 & 2.4 & 4.3 \\
\hline & 13 & 4.9 & 3.2 & 2.5 & 4.5 & 4.6 & 5.5 \\
\hline & 17 & 5.9 & 4.2 & 3.6 & 5.0 & 5.2 & 6.6 \\
\hline & 21 & 7.2 & 5.2 & 4.7 & 6.4 & 6.3 & 7.6 \\
\hline & 25 & 4.9 & 6.0 & 5.2 & 6.9 & 7.3 & 8.7 \\
\hline \multirow{5}{*}{$\begin{array}{l}\text { Ne atom density HF/6- } \\
\quad 311 \mathrm{G}\end{array}$} & 9 & 3.6 & 3.1 & 2.0 & 2.6 & 2.5 & 3.7 \\
\hline & 13 & 3.8 & 3.9 & 2.5 & 3.6 & 3.6 & 3.7 \\
\hline & 17 & 4.6 & 3.7 & 3.7 & 3.6 & 3.6 & 4.5 \\
\hline & 21 & 4.2 & 5.2 & 4.1 & 4.0 & 4.2 & 4.7 \\
\hline & 25 & 4.5 & 6.2 & 4.8 & 5.1 & 5.4 & 5.7 \\
\hline \multirow{5}{*}{$\begin{array}{l}\text { Ar atom density HF/6- } \\
\quad 311 \mathrm{G}\end{array}$} & 9 & 2.0 & 2.6 & 2.9 & 2.4 & 3.1 & 2.8 \\
\hline & 13 & 3.3 & 3.2 & 2.7 & 3.7 & 3.5 & 3.6 \\
\hline & 17 & 3.9 & 4.2 & 3.4 & 3.5 & 3.5 & 4.2 \\
\hline & 21 & 4.6 & 4.5 & 3.9 & 4.8 & 4.3 & 4.6 \\
\hline & 25 & 4.3 & 4.9 & 4.8 & 4.9 & 4.3 & 5.3 \\
\hline \multirow[t]{5}{*}{$1 /\left(1+r^{4}\right)$} & 9 & 0.8 & 2.5 & 2.6 & 1.1 & 1.2 & 0.9 \\
\hline & 13 & 0.8 & 2.7 & 3.4 & 1.1 & 1.3 & 1.0 \\
\hline & 17 & 0.8 & 2.9 & 3.4 & 1.2 & 1.3 & 1.0 \\
\hline & 21 & 0.8 & 3.1 & 3.5 & 1.2 & 1.3 & 1.1 \\
\hline & 25 & 0.8 & 3.3 & 3.7 & 1.2 & 1.4 & 1.1 \\
\hline
\end{tabular}

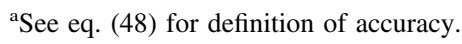

below. They are included, not because they are conclusive, but because they are interesting.

The results in Table 5 were obtained using the standardized roots and weights of Tables 2 and 3 . In each case, we measure the quality of a quadrature estimation ("Approx") of an integral by comparing it with the exact value ("Exact") using

$$
\text { Accuracy }=-\log _{10}\left|\frac{\text { Approx }}{\text { Exact }}-1\right|
$$

The resulting values are essentially the number of correct digits in the quadrature result.

The first $f(r)$ that we tested was a Gaussian with unit exponent. Although this is a simple and important function, it is not treated exactly by any of the radial quadratures that this article has considered. (We note that two-point Hermite quadrature ${ }^{8}$ would be exact.) For this integrand, Table 5 reveals that the grids may be ranked as
Handy $<$ Becke $<$ Ahlrichs $\approx$ Knowles

$$
<\text { MultiExp }<\text { Laguerre }
$$

We then explored the effect of adding a second, tighter Gaussian. This improves the Handy accuracy and worsens the Ahlrichs and Laguerre accuracies, yielding the ranking

Handy $\approx$ Becke $<$ Ahlrichs $<$ Knowles

$$
<\text { Laguerre }<\text { MultiExp }
$$

Adding a third, even tighter Gaussian to $f(r)$ leads to deterioration in the Ahlrichs, Knowles, Laguerre, and MultiExp accuracies and narrows the spread of the six grids to yield

Handy $<$ Ahlrichs $\approx$ Knowles $<$ Becke

$$
<\text { Laguerre }<\text { MultiExp }
$$


These first three examples are artificial so we next consider more realistic $f(r)$ functions.

To this end, we have selected the first three inert gas atoms (He, $\mathrm{Ne}$, and Ar) and have used quadrature to estimate the electron count integral:

$$
\int_{0}^{\infty} 4 \pi r^{2} \rho(r) d r=N
$$

where $\rho(r)$ is an electron density and $N$ is the total number of electrons. The density of the He atom is a monotonically decreasing function of $r$ and is well approximated by a linear combination of exponential functions. It is therefore not surprising to find that the MultiExp grids are the most accurate of the six. Table 5 shows that the grid ranking in this case is

Handy $<$ Becke $<$ Ahlrichs $\approx$ Knowles

$$
<\text { Laguerre }<\text { MultiExp }
$$

but we note that the 25-point Laguerre grid performs surprisingly poorly. The density of the $\mathrm{Ne}$ atom contains one component that decays rapidly (due to the core electrons) and another that decays more slowly (due to the valence electrons). This multiscale behavior reduces the accuracy of most of the grids, but not Becke's. The grid ranking here is

$$
\begin{aligned}
\text { Handy }<\text { Laguerre } \approx \text { Ahlrichs } \approx & \text { Knowles } \\
& <\text { MultiExp } \approx \text { Becke }
\end{aligned}
$$

The density of the Ar atom has three components, the innermost of which decays very rapidly and, as before, this tends to lower the accuracy of the grids. Although it is no longer possible to rank them unambiguously, we note that the MultiExp grid remains competitive.

Finally, we sound a note of caution. Any quadrature scheme, if applied uncritically to an inappropriate integrand, can yield spectacularly bad results. To illustrate this, we have used each of the six grids to estimate the slowly converging radial integral

$$
\int_{0}^{\infty} \frac{r^{2}}{1+r^{4}} d r=\frac{\pi}{2 \sqrt{2}}
$$

and the results are shown in the final rows of Table 5. The grid ranking

Laguerre $<$ MultiExp $<$ Ahlrichs $<$ Knowles $<$ Becke $<$ Handy

is the reverse of that obtained when integrating a single Gaussian and reflects the size of the outermost root (Table 2). The Becke and
Handy grids (which sample the integrand at large $r$ ) perform much better than the relatively compact grids, which struggle to obtain even one significant digit. We do not recommend the MultiExp grid for such integrals.

\section{Concluding Remarks}

Radial integrals are ubiquitous in chemical physics and are frequently estimated using quadrature. We have reviewed a number of radial quadrature schemes and have introduced a new one (MultiExp) that is explicitly constructed to be exact when the non-Jacobian part of the integrand is a linear combination of geometrically spaced exponential functions. The roots and weights of the new scheme are given in eqs. (34) and (35), and the associated "log-squared" roots and weights are listed in Table 1. Preliminary numerical investigations suggest that the new radial quadrature scheme may be useful in physical applications. We are constructing a new standard grid (SG-0) for use in density functional theory, and the radial part of this will use MultiExp quadrature. We will publish this elsewhere. ${ }^{19}$

\section{Acknowledgments}

We thank Dr. Don Rees and Prof. Peter Knowles for useful discussions.

\section{References}

1. Sobolev, S. L. Sibirsk Mat Zh 1962, 3, 769.

2. McLaren, A. D. Math Comput 1963, 17, 361.

3. Stroud, A. H. Approximate Calculation of Multiple Integrals, PrenticeHall: Englewood Cliffs, 1971; pp 294-303.

4. Lebedev, V. I. Sibirsk Mat Zh 1977, 18, 132.

5. Konyaev, S. I. Mat Zametki 1979, 25, 629.

6. Delley, B. J Comput Chem 1996, 17, 1152.

7. The scale factor $R$ is arbitrary, but the best accuracy is achieved if $R$ reflects the domain of $r$ over which $f(r)$ is large.

8. Handbook of Mathematical Functions. Abramowitz, M., Stegun, I. E., Eds.; Dover: New York, 1970.

9. Becke, A. D. J Chem Phys 1988, 88, 2547.

10. Murray, C. W.; Handy, N. C.; Laming, G. J. Mol Phys 1993, 78, 997.

11. Treutler, O.; Ahlrichs, R. J Chem Phys 1995, 102, 346.

12. Mura, M. E.; Knowles, P. J. J Chem Phys 1996, 104, 9848.

13. We note the weight function $w(x)=\ln x$ is mentioned on p. 890 of ref. 8 .

14. Szegö, G. Orthogonal Polynomials, American Mathematical Society: New York, 1959.

15. For example, the first three are: $Q_{0}(x)=1, Q_{1}(x)=8 x-1$, $Q_{2}(x)=7992 x^{2}-4104 x+217$.

16. However, see Yang, W. J Chem Phys 1991, 94, 1208.

17. Parr, R. G.; Yang, W. Density-functional theory of atoms and molecules, Oxford: New York, 1989.

18. Wang, W.-P.; Parr, R. G. Phys Rev A 1977, 16, 891.

19. Chien, S.-H.; Gill, P. M. W. In preparation. 